\title{
N-path filters and Mixer-First Receivers: A Review
}

\author{
Eric A.M. Klumperink, Hugo J. Westerveld, Bram Nauta \\ University of Twente, CTIT, IC Design group, Enschede, The Netherlands
}

\begin{abstract}
To realize a Software Defined Radio covering the mainstream 0.5-6 GHz wireless communication bands, new SAW-less radio receiver architectures are being explored which realize selectivity in a more flexible and programmable fashion. $\mathrm{N}$-path filters and mixer-first receivers can offer high-linearity high-Q RF-filtering around a center frequency defined by a digital clock, which offers the desired flexible programmability. This paper reviews recent research on $\mathrm{N}$-path filters and mixerfirst receivers, identifies advances in performance analysis, circuit performance and applications.
\end{abstract}

Keywords-Mixer-first reeceiver, $N$-path filter, Frequency Translated filtering, passive mixer, CMOS, switch-R-C circuit, software defined radio, reconfigurable radio, $R F$, wireless

\section{INTRODUCTION}

In a world with ever more mutually interfering wireless devices, selectivity by high-Q filtering is of paramount importance. Traditionally, this filtering is implemented by high-Q LC-filters or devices that can be modelled as such (e.g. devices exploiting transmission lines, Surface Acoustic Waves (SAW) or Bulk Acoustic Waves (BAW)). Although these devices can achieve very good selectivity and excellent linearity, they lack the programmability wanted for software defined radio. Moreover, compared to solutions exploiting CMOS IC technology, size and cost are key issues.

CMOS IC technology is very suitable to realize wellmatched highly linear oxide-capacitors, while Moore's law also offers faster digital circuits and better switches in terms of low on-resistance and parasitic capacitance. As N-path filters exploit switches and capacitors, see e.g. Fig. 1, while offering high-Q filteing around a digitally programmable switching frequency, this explains the recent interest in $\mathrm{N}$-path filters and mixer-first receivers for software defined radio.

Historically, different names have been used like "Narrow Band-Pass filter Using Modulation" [1], "Commutated Network"[2], "Comb Filter using Commutated Capacitors" [2, 3], "Sampled-Data Band-Pass filter" [4], Frequency-Translated Filter [4], "N-Path filter" [5]. We will use the generalized term $\mathrm{N}$-path filter here, as in the filter survey by Moschytz [6]: "N identical networks $\mathrm{H}(\mathrm{j} \omega)$ are cyclically switched into the signal path. Thus, the total N-phase impulse network has timevariable characteristics. If a low-pass filter is used to provide the $\mathrm{H}(\mathrm{j} \omega)$ function in each of the paths, the cyclical switching process causes a low-pass to bandpass transformation." Note that we do not assume a uni-lateral transfer for each of the $\mathrm{N}$ signal paths, although this is sometimes convenient to simplify analysis, e.g. as in [5].

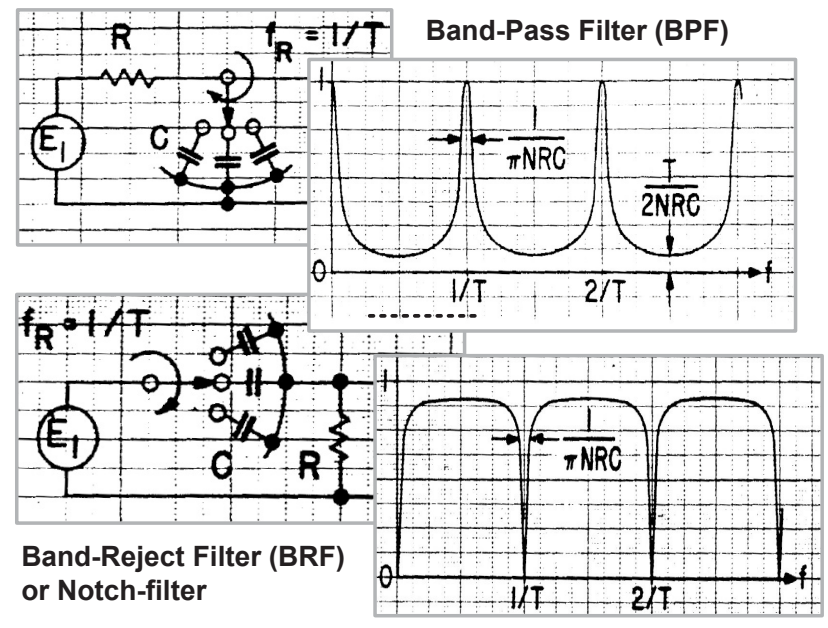

Fig. 1. Commutated band-pass and band-stop filter and their frequency response as predicted by Smith in 1953 [2]. Early commutators were implemented as motor driven rotating mechanical switches.

This paper reviews the literature on N-path filters and mixer-first receivers, trying to identify advances in performance analysis (section II), steps in the evolution of mixer-first receivers (section III) and N-path filters (section IV), concepts to lower power dissipation (section V) and new applications (section V). The primary focus will be on high linearity passive-first frequency-selective switch-R-C circuits. Active feedback techniques as reviewed in [7] and wideband approaches like in [8] are not covered, but can be found in other reviews like [9-11]. The reference list focusses on journal papers, even if earlier conference papers exist.

\section{PERFORMANCE ANALYSIS OF N-PATH FILTERS}

Although the switch-R-C circuits in Fig. 1 look simple, mathematical analysis of their transfer function is complicated by their time-variant nature combined with the memory effect of the capacitors. We will describe different simplifications that have been used over time to make performance analysis tractable.

Early papers [2, 3, 5] make simplifying assumptions ultimately leading to a kind of "sampled data" approximation, hence the title "Sampled Data Band-Pass Filter" [4]. Assuming such ideal sampling there is no loss and this leads to an interesting analogy of the commutator in Fig. 1 with a transmission line: if the rotator cycle-time is equal to the period of a sinewave input signal, the voltages on the capacitors become samples of one period of the sinewave. 

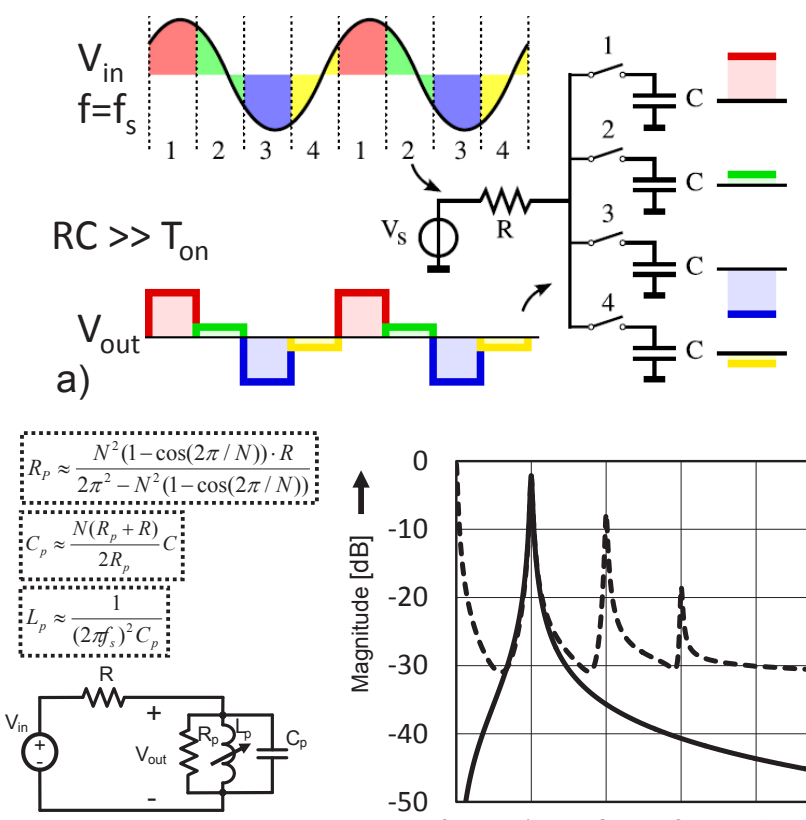

b)

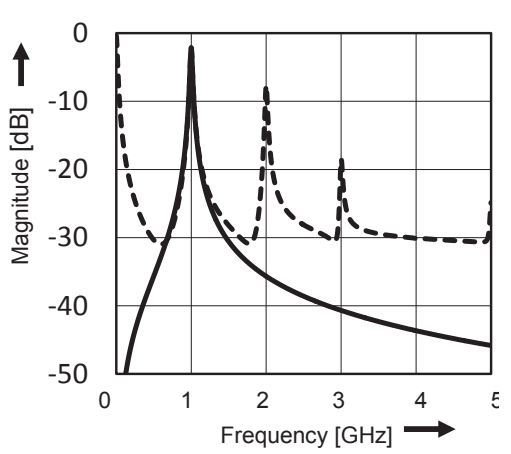

Fig. 2. 4-path switch-R-C filter: a) time-domain waveforms; b) Simulated transfer function (dashed line) and approximate R-L-C model (drawn) [12] $(\mathrm{R}=50 \Omega, \mathrm{C}=50 \mathrm{pF}, 1 \Omega$ switch resistance, $1 \mathrm{GHz}$ clock with $25 \%$ duty cycle).

After one full commutator cycle, the new incoming sinewave is hence "confronted with" a sample stored on the capacitor one period ago. This behavior resembles that of an open transmission line of length $\mathrm{T} / 2$, which fully reflects the input signal of one period ago $[2,3]$. As a result, the transfer function shown in Fig. 1 has the well-known comb filter behavior of transmission line filters, with identical gain for all harmonics. Although the identical gain at harmonics is a somewhat crude approximation (see Fig. 2 for $\mathrm{N}=4$ [12]), it gives useful first order insight. Moreover, [2, 3] already rather accurately estimated the $-3 \mathrm{~dB}$ bandwidth of the filter:

$$
f_{-3 d B}=\frac{1}{\pi N R C}
$$

This means that the bandwidth is inversely proportional to the total capacitance $N C$. Essentially the N-path filter downconverts the RF-signal, low-pass filters it, and then up-converts it again to RF (the switches in Fig. 1 serve both the down- and up-conversion purpose). Hence the low-pass filter characteristic is simply shifted around the commutatorfrequency. Intuitively, the RF-bandwidth is twice that of an RC-lowpass filter, multiplied by $\mathrm{N}$ as each capacitor "sees" the resistor R only for a duty cycle $1 / \mathrm{N}$.

From this simple analysis we can gain some very relevant insights explaining the interest in N-path filters for software defined radio: 1) the center frequency of the filter is accurate and widely programmable using digital clocking; 2) the RFbandpass roll-off is essentially the same as for a real-pole RC low-pass filter, but frequency shifted; 3) the equivalent $Q=$ $f_{s} / f_{-3 d B}$ can approach SAW-filter Q-values, as GHz switching frequencies are feasible in nanometer CMOS, while $f_{-3 d B}$ can be in the low MHz region if we add sufficient capacitance; 4)
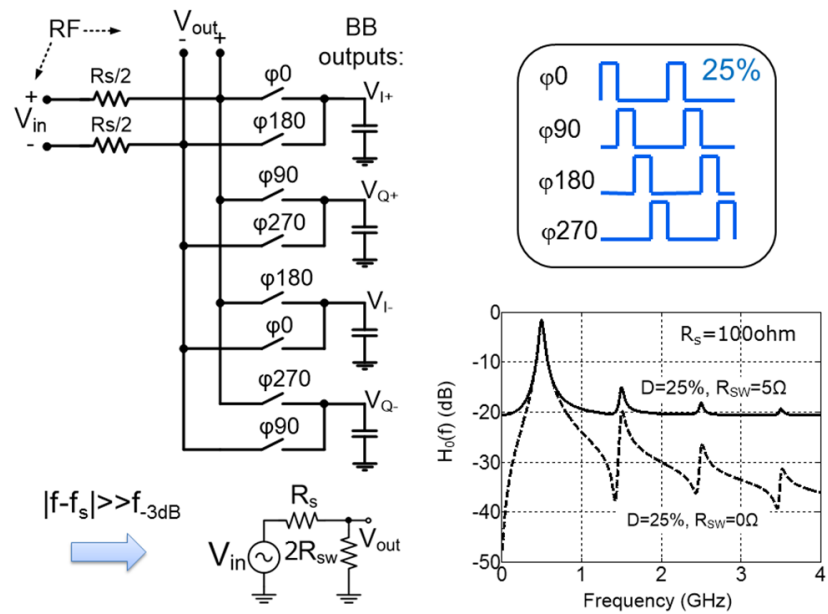

Fig. 3. 4-Path mixer-first receiver with $25 \%$ duty-cycle clocks, differential RF-input $\mathrm{V}_{\text {in }}$, low-pass baseband quadratures outputs $\mathrm{V}_{\mathrm{I}} \mathrm{V}_{\mathrm{I}-} \mathrm{V}_{\mathrm{Q}+} \mathrm{V}_{\mathrm{Q}-}$ and band-pass filtered RF-output $\mathrm{V}_{\text {out }}$. Even harmonic responses cancel. Switch resistance limits out-of-band filter rejection, as modelled via $R_{s}$ and $2 R_{s w}$.

linearity perspectives are good as MOSFET passive mixers can have very good linearity.

The early N-path filters use the output signal in a timecontinuous fashion. In the end of the 60 's research attention gradually shifted to time discrete signal processing, where the output signal is only interpreted at certain instants. To achieve a sharper filter roll-off, complex pole pairs are wanted. This was implemented combining switched capacitor networks first with finite gain amplifiers [13] and later with high-gain Opamps [6]. Although N-path filters with time-continuous output are still mentioned in the filter survey of [6] and in 70s textbooks on switched-capacitor filters, literature references fades out in the $80 \mathrm{~s}$. In papers on N-path filters that we did find, the filter output is interpreted in a time discrete manner, as is apparent from the z-domain analysis that is used [14]. Only in the last decade N-path filters with a time-continuous output regained research interest [15-25]. Moreover, mixer-first receivers exploiting N-path frequency translated filtering were proposed [26-32]. Fig. 3 shows a mixer with differential RFinput exploiting two sets of switches driven by a $25 \%$ duty cycle. Capacitor baseband voltages $\mathrm{V}_{\mathrm{I}^{+}}, \mathrm{V}_{\mathrm{Q}^{+}}, \mathrm{V}_{\mathrm{I}}$-, $\mathrm{V}_{\mathrm{Q}-\text { are now }}$ used as output instead of the RF voltage $V_{\text {out }}$. Note however that RF filtering still takes place, as the switches not only down-convert to baseband, but also up-convert so that $\mathrm{N}$-path filtering occurs at the RF-input.

This review focusses on N-path switch-R-C circuits with a time-continuous output. In contrast to circuits with a time discrete output that use sampling with fast settling, timecontinuous N-path filters with good frequency selectivity show extremely slow settling, analogous to high-Q passive filters. We can actually define two regions for a simple switch-R-C circuit with on-time $\mathrm{T}_{\text {on }}[16,33,34]$ :

$$
\begin{array}{ll}
\text { Sampling region: } & T_{\text {on }} \gg 2 R C \\
\text { Mixing region: } & T_{\text {on }} \ll 2 R C
\end{array}
$$

Using a simplified analysis based on energy conservation while assuming very big RC times, Cook et al [26] found .375 


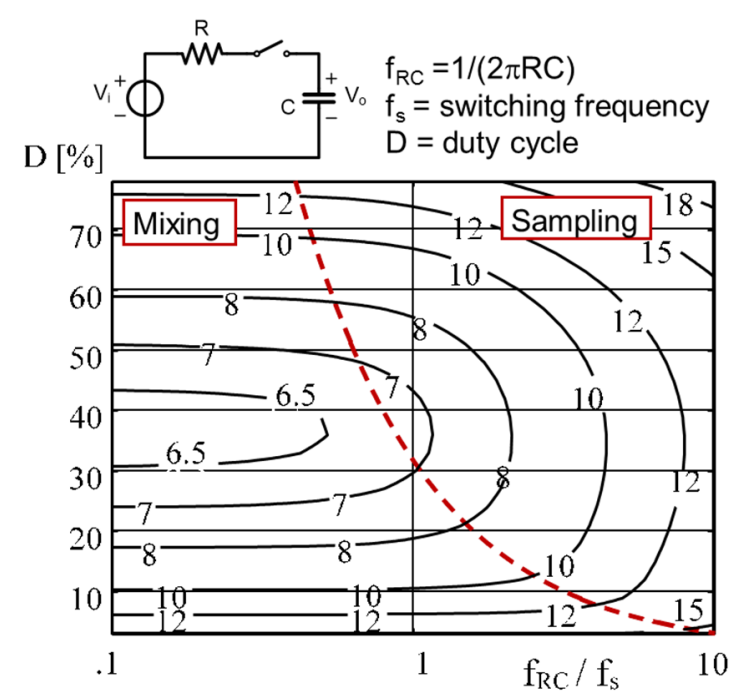

Fig. 4. Constant Noise Figure contours for a switch-R-C down-conversion mixer for arbitary RC-time, switching frequency and duty cycle. Note the lower noise figure in the mixing-region for big $\mathrm{RC}$ due to less noise folding.

as best achievable noise figure for a 4-path mixer with $25 \%$ duty cycle in each path.

This result was generalized and confirmed by the unified analysis of samplers and mixers in [16]. In Fig. 4 [35], noise figure of a switch-R-C circuit is plotted for arbitrary values of switching frequency, RC-time and duty cycle. The theoretical noise figure for a 4-path mixer is $6.9 \mathrm{~dB}$. When combining 4 output signals, SNR is improved $4 \mathrm{x}(6 \mathrm{~dB})$, leading to a theoretical minimum noise figure of $0.9 \mathrm{~dB}$, fitting to the $\pi^{2} / 8[26]$. This demonstrates the low-noise perspective of $\mathrm{N}$ path filters and mixers.

Using the analysis procedure in [16], N-path filter transfer has been analyzed in [12] and the equivalent R-L-C filter model of Fig. 3 was derived for arbitrary $\mathrm{N}$ to fit the fundamental response. The analysis was also used to find the transfer function for N-path notch filters [23], gain-boosted N-path filters [36], and an N-path filter preceded by a series-inductor to realize time-continuous low-pass pre-filtering [37]. Such pre-filtering is important, as N-path filters show folding of RFsignals around some harmonics of the clock-frequency. The first folding frequency band is around $(N-1) f_{s}$, similar to aliasing in discrete time circuits, albeit with more attenuation. Using more paths, e.g. 8 instead of 4 , allows for cancelling more folding products. The analysis of $[16,17,23]$ is suitable to analyze folding, as no assumptions about the input frequency are made. Whereas this unified analysis of mixing and sampling (see Fig. 4) analyzes the continuous time output, a recent unified analysis [33, 34] focusses on a time-discrete output. After some simplifying assumptions on reconstruction filtering, a time-continuous output can be calculated, arriving at the same result as [16]. Recently, conversion matrices were also proposed to reproduce and extend some earlier N-path analysis results [38]. Still, all these analysis procedures are rather cumbersome, so that it may be beneficial to make a priori assumptions that simplify analysis, while still providing a good approximation, albeit with a more limited scope.

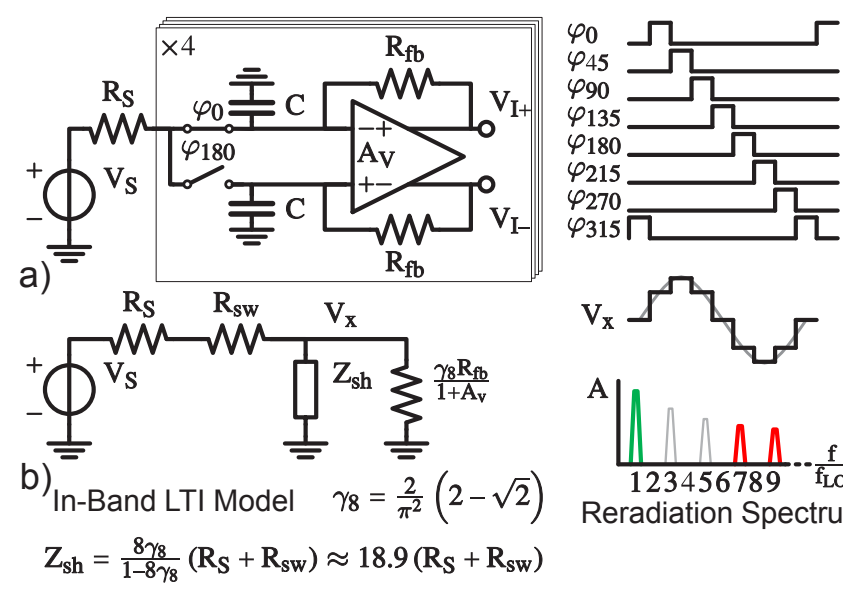

Fig. 5. a) 8-path mixer-first receiver [28, 29]; b) in-band LTI model.

The transfer function and input impedance of the circuit in Fig. 2a for a sinewave voltage input can be approximated assuming $\mathrm{RC}>>\mathrm{T}_{\text {on }}$ and a virtually constant voltage on the capacitors [28-31, 39]. The difference between the sinewave input $V_{\text {in }}$ and the constant capacitor voltage $V_{\text {out }}$ is now across resistor $\mathrm{R}$, and the average power dissipation can be evaluated. This leads to a reduction in amplitude related to $\operatorname{sinc}(\pi / N)$. In $[28,29]$ this dissipation is modelled in an LTI baseband model by an equivalent impedance $Z_{\text {sh }}$ (see Fig. 5 b). Baseband resistance in parallel to $C$, e.g. $R_{\mathrm{fb}} /\left(1+\mathrm{A}_{\mathrm{v}}\right)$ in Fig. 5a, resulting in an extra loss resistance. This LTI model is extended in [39] to derive design equations for important properties of a mixerfirst receiver, and the relation to the number of paths/phases.

Another analysis method assumes a current driven mixer with $25 \%$ duty cycle driving four baseband lowpass impedances $Z_{\mathrm{BB}}(\mathrm{f})[20,40]$. The $\mathrm{RF}$ source is now modelled as a current source with parallel impedance $Z_{\mathrm{s}}\left(\mathrm{f}_{\mathrm{RF}}\right)$. It is assumed that the RF-input frequency $f_{R F}$ is confined to $f_{s} / 2$ and $3 f_{s} / 2$, and the current entering the switched 4-path filter is approximated by a Fourier series. An approximation of the RF input voltage in the form of an infinite sum is found where not only $Z_{\mathrm{s}}$ around $\mathrm{f}_{\mathrm{RF}} \approx \mathrm{f}_{\mathrm{s}}$ matters, but also around $\mathrm{f}_{\mathrm{RF}} \approx(4 \mathrm{k}+1) \mathrm{f}_{\mathrm{s}}$. In other words: the source impedance $Z_{\mathrm{s}}$ "seen by the switched mixer" at harmonics of the switching frequency is important. Also, imperfections like clock mismatches and phase noise are analyzed in [20]. However, due to the input frequency limitation, signal folding from frequencies higher than $3 \mathrm{fs} / 2$ cannot be analyzed in contrast to [16].

\section{MiXer-FIRST EVOLUTION}

In contrast to traditional radio receivers, mixer-first receivers lack a Low Noise Amplifier (LNA) before the mixer. One of the early mixer-first receivers mainly removes the LNA to save power [26]. The quadrature receiver with $25 \%$ duty cycle achieves $<6 \mathrm{~dB}$ Noise Figure and IIP $3=-7.5 \mathrm{dBm}$ at only $720 \mu \mathrm{W}$ power consumption.

Another motivation to remove the LNA is spurious free dynamic range (SFDR), which is determined by both noise figure and IIP3 [27]. As receiver noise figure is often only a 


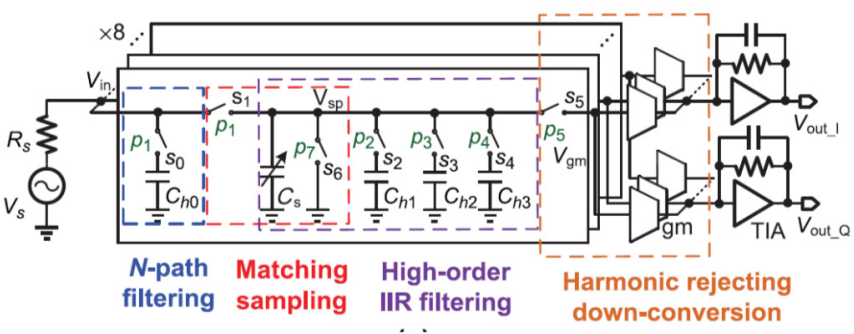

Fig. 6. N-Path filtering enhanced by discrete time switched-capacitor chargedomain $3^{\text {rd }}$ order Infinite Impulse Response (“3IIR") filtering [41].

few $\mathrm{dB}$ above the fundamental $0 \mathrm{~dB}$ limit, there is more room at the top of the dynamic range by improving IIP3. As passive mixers typically have $>20 \mathrm{~dB}$ better IIP3 than LNAs, while opamps can realize high linearity in baseband, it makes sense to remove the LNA [27]. An in-band linearity of $+11 \mathrm{dBm}$ was achieved with $\mathrm{NF}<6.5 \mathrm{~dB}$ at $65 \mathrm{~mW}$ rendering $\mathrm{SFDR}>79 \mathrm{~dB}$ in $1 \mathrm{MHz}$ bandwidth, which compares favorably in SFDR to professional spectrum analyzers.

Probably the most influential papers on mixer-first receivers are from Molnar's group [28, 29], presenting several useful concepts (see Fig. 5a):

1) Input impedance matching is provided combining switched-C loss, switch resistance and the input impedance of the baseband amplifier, allowing for a NF only slightly higher than $3 \mathrm{~dB}$.

2) 8-phase mixing, instead of 4-phase is used so that the filtered staircase approximation of the RF signal contains fewer harmonics. This lowers NF, harmonic re-radiation and harmonic downconversion (see also [30, 39]). LO-radiation reduction by calibration is discussed in $[42,43]$.

3) The baseband amplifiers contain tunable feedback resistors connected between the I and Q channels (not shown in Fig. 5a). With this complex feedback, a non-real input impedance can be realized at the RF port, allowing for matching to complex antenna impedances and compensation of input parasitics, resulting in an $\mathrm{S}_{11}$ notch that can be tuned to the LO frequency $[28,30]$.

With the techniques discussed above, IIP $3>25 \mathrm{dBm}$ and blocker compression $\mathrm{B} 1 \mathrm{~dB}>0 \mathrm{dBm}$ is feasible for high offset frequencies. Still, SAW filters can achieve significantly higher values at smaller offset frequencies. Hence, alternative techniques to improve linearity and blocker filtering further have been explored. One example combines N-path filtering with switched-capacitor Infinite Impulse Response (IIR) charge domain filtering (see Fig. 6). The higher-order IIRfiltering renders steeper filtering and better blocker rejection rendering a $\mathrm{B} 1 \mathrm{~dB} \approx 15 \mathrm{dBm}$ at $30 \mathrm{MHz}$ offset, albeit at $7-10 \mathrm{~dB}$ Noise figure [41]).

Another passive pre-filter approach targeting extreme linearity exploits cross-coupling of a switched-R mixer with a switch-R-C N-path notch filter as shown in Fig. 7 [44]. Close to the switching frequency, the notch-filter is high-ohmic and signal is passed. However, the cross-coupled path is low-ohmic for out-of-band blockers, circulating blocker current back to the RF-source (see Fig. 7), so that it does not "reach" the TIA, leading to an IIP3 as high as $+42 \mathrm{dBm}$ [44].

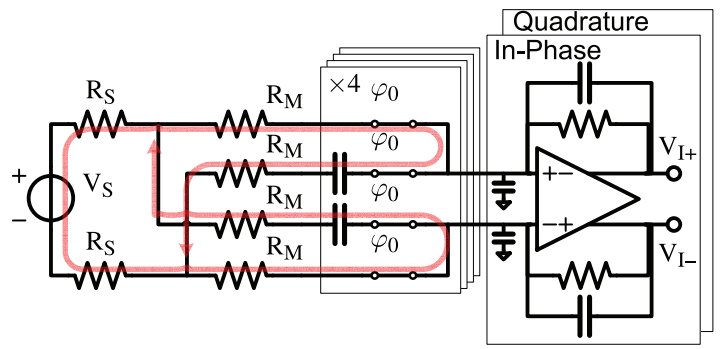

Fig. 7. Switched-R mixer with differential RF-input, with a cross-coupled switch-RC path for blocker by-passing. Due to the symmetry and highlinearity, an IIP $3>+42 \mathrm{dBm}$ and $\mathrm{B} 1 \mathrm{~dB}>11 \mathrm{dBm}$ is achieved [44].

Mixer-first receivers often realize very good linearity, but usually at a noise figure in the range of $3-6 \mathrm{~dB}$ for good impedance matching. However, it is possible to go below $3 \mathrm{~dB}$ adding controlled positive feedback to help realize matching [45]. Also, combining low switch resistance with careful transimpedance amplifier design, $2.6 \mathrm{~dB}$ Noise figure has been demonstrated [43].

\section{N-PATH FILTER EVOLUTION}

One of the early papers proposing $>100 \mathrm{MHz}$ RF-frequency $\mathrm{N}$-path filters with a time-continuous output is [15]. It demonstrates a single-ended 8-path bandpass filter driven by a CMOS ring oscillator, with high quality factor of up to 300 , while realizing $240-530 \mathrm{MHz}$ tuning range. IIP3 and out-ofband blocking results are not reported.

In our group we (re-)invented the switched-capacitor bandpass filters and only discovered older work during publication attempts. Triggered by good linearity results of [27], we explored a fully differential implemented 4-path filter, focusing on programmability and linearity. A tuning range of $100 \mathrm{MHz}$ $-1.2 \mathrm{GHz}$ was demonstrated with IIP3 $>18 \mathrm{dBm}$ and $2 \mathrm{dBm}$ 1dB-compression point [23]. Notch filters, both with a differential and single input, were also evaluated in $65 \mathrm{~nm}$ and analyzed in-depth [23].

Mirzaei et al exploited N-path filters in $[20,46]$ to realize a quad-band SAW-less receiver exploiting to meet all GSM/EDGE requirements, in particular the $15 \mathrm{~dB}$ Blocker noise figure requirements for a $0 \mathrm{dBm}$ blocker at $80 \mathrm{MHz}$ offset frequency. It achieves $>+2 \mathrm{dBm}$ out-of-band $1 \mathrm{~dB}$ blocker compression. As discussed in the previous section, the work in $[20,46]$ also greatly contributed to analysis.

In [19] the use of N-path filters to realize filtering in a superheterodyne receiver architecture is explored, with a passband at the difference of two clock frequencies. Using $110 \mathrm{MHz}$ IF-frequency, $2.8 \mathrm{~dB}$ Noise Figure and $-8 \mathrm{dBm}$ IIP3 is achieved. Image rejection and harmonic rejection is crucial in heterodyne receivers, as explored further in [47]. A flexible Weaver architecture augmented with harmonic rejection was proposed, with a tunable IF-frequency between 100 and $500 \mathrm{MHz}$. As image rejection and harmonic rejection for some conversions is limited to $20-45 \mathrm{~dB}$, additional pre-filtering is required for most practical applications.

Ideally, capacitors shunt all out-of-band signal to ground. However, switch resistance limits the conductance to ground, 


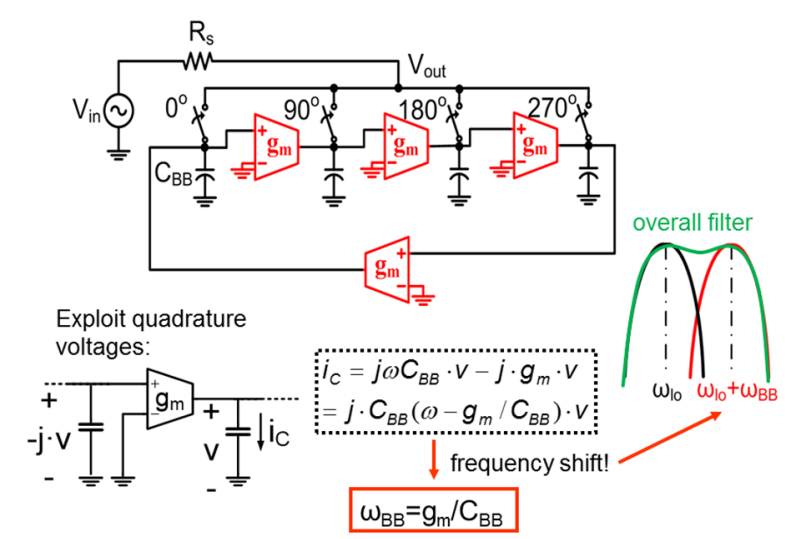

Fig. 8. Improving filter shape by transconductor coupling between nodes with quadrature signals shift [24]

and hence filter attenuation. The achievable attenuation can be estimated with a simple resistive divider model as shown in Fig. 3. Using a second set of switches that sense the capacitor voltages can largely eliminate the problem [22, 47].

Another limitation of N-path filters is their "rounded filter shape" associated with the real poles. This can be improved as shown in Fig. 8, if two N-path filters with shifted center frequencies are used [22]. This shift is implemented by transconductors connected between baseband nodes with 90 degrees phase shift, contributing a gm/C shift in frequency (see Fig. 8). However, baseband transconductors add 1/f noise, while this problem does not exist if transconductors are used at RF. To approximate the ideal brick-wall filter shape closer, it would be desired to couple multiple stages of high-Q resonators. This cannot be done directly with switched capacitor N-path filter sections, as putting them in parallel simply results in a second order R-L-C with reduced bandwidth (more C). As suggested by Heinlein [48], it is possible to couple N-path band-pass filter sections by gyrators. Fig. 9 shows the block schematic of an 8-path 6-order band-pass filter [24], with a fully passive first section for high linearity. A nice flat-top band-pass filter shape is realized, with $19 \mathrm{~dB}$ gain and $3 \mathrm{~dB}$ Noise Figure. The filter has $8 \mathrm{MHz}$ bandwidth and shows an out-of-band IIP3 of $+26 \mathrm{dBm}$ and compression point of $+7 \mathrm{dBm}$ at $50 \mathrm{MHz}$ frequency offset, tunable from $100 \mathrm{MHz}$ to $1.2 \mathrm{GHz}$.

In order to increase flexibility for software defined radio, reconfigurable N-path filters have also been explored. In [49] both reconfigurable band-pass and band-reject filtering is demonstrated, while the gm-C shift technique from Fig. 8 is used to allow for continuous shifts of the N-path centerfrequency with up to $100 \mathrm{MHz}$.

Other work focusses on improving N-path filter loss and out-of-band rejection, by putting N-path filter sections between inductors, like in a synthetic transmission-line [50]. This tunes out capacitive parasitics, reducing loss and noise, while also reducing folding (see [37]). Other work in advanced SOI technology demonstrates improved linearity and increased frequency range [51]. Moreover, [52] demonstrates that harmonic folding can be limited to $<-60 \mathrm{dBc}$ using a 16-path band-pass filter.
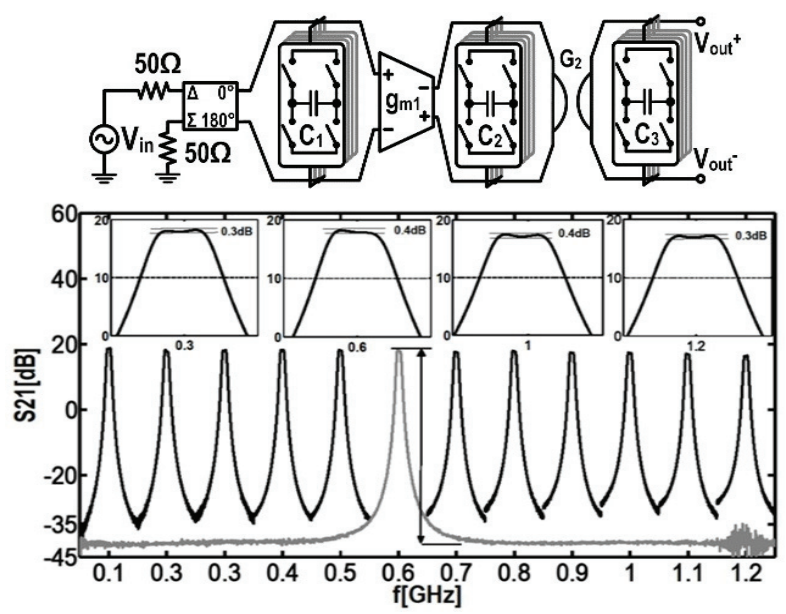

Fig. 9. Sixth-order band-pass-filter-LNA with one passive 8-path BPFsection and two others coupled by a transconductor and gyrator [24].

\section{REDUCING POWER CONSUMPTION}

$\mathrm{N}$-path filter bandwidth is inversely proportional to the NRC product (see eqn.(1)), so if the filter is operating in a $50 \mathrm{ohm}$ environment a big total capacitance is required to realize a narrow bandwidth (e.g. $32 \mathrm{nF}$ for $200 \mathrm{kHz}$ GSMbandwidth [54]). Switch resistance should be much smaller than source resistance $\mathrm{R}$ to minimize noise and achieve good filter attenuation (see Fig. 3). However, lowering switch resistance requires wider transistors with larger gate capacitance and hence more dynamic power consumption.

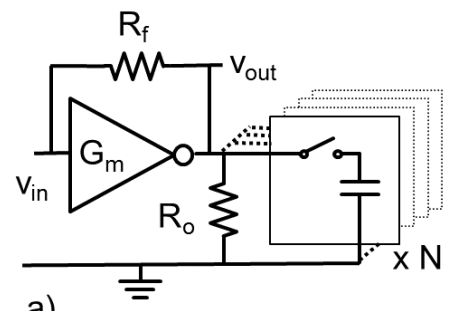

a)

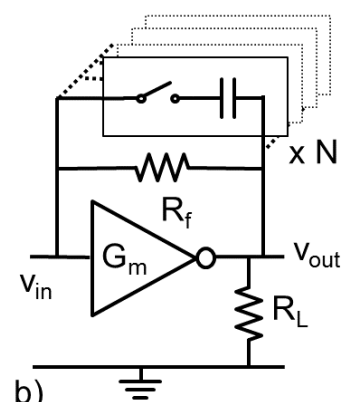

b)
Fig. 10. Increasing the N-path impedance level by: a) Exploiting a Transconductor with load resistance $\mathrm{R}_{0}>\mathrm{R}$ [53]; b) Exploiting the Miller effect ("Miller Band-Pass Filter" or "Gain-boosted N-path filter") [36, 54].

Hence attempts have been made to increase the impedance level by combining an N-path filter with an LNA or LNTA, as shown in Fig. 10. In $[53,55,56]$ an LNTA which can be modelled as a transconductance $G_{m}$ and output resistance $R_{o}$ precedes the N-path filter, where mainly output resistance $R_{o}$ defines the $\mathrm{R}$ seen by the switched-capacitor $\mathrm{N}$-path filter as shown Fig. 10a (assuming $\mathrm{R}_{\mathrm{f}}$ is high for self-biasing). If downconversion is wanted anyway, the baseband voltages can directly be used as output, while the LNA output voltage is filtered by N-path filtering, improving interference robustness. The output resistance $R_{o}$ of the LNTA can be significantly higher than $50 \mathrm{ohm}$. If it is e.g. 10x higher, this leads to $10 \mathrm{x}$ lower capacitance for the same bandwidth, 10x higher allowed switch-resistance and hence 10x lower dynamic power 


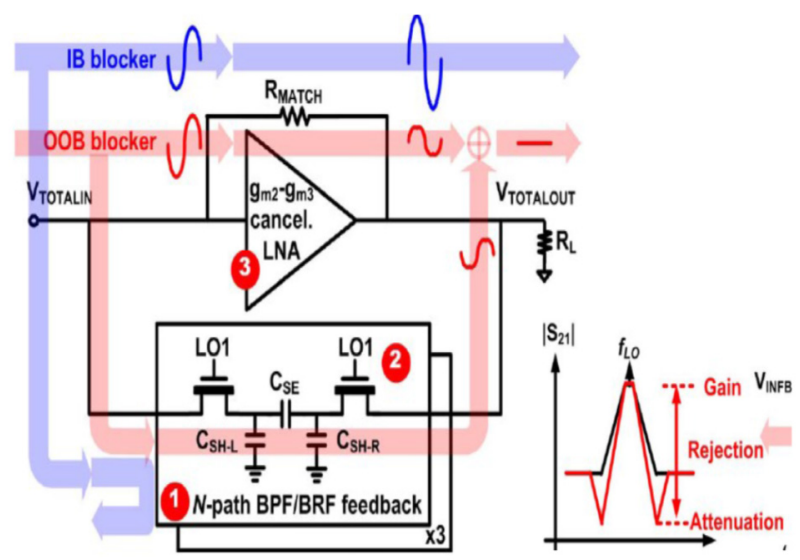

Fig. 11. LNA with N-path filter in the feedback path consisting of a series capacitor realizing a notch filter [23], and grounded shunt capacitors that realize the attenuation notches in the overall transfer function [60].

consumption. As blockers are now amplified in the LNA despite of the N-path filtering, a high supply voltage may be desired in the LNA to allow for enough voltage swing without clipping [53]. Another motivation for this LNA-first architecture followed by $\mathrm{N}$-path filtering is lower noise figure [57]. To maximize the output resistance and still cope with strong out-of-band blockers, an LNTA can also be used as has been proposed in $[55,56]$. Now the parasitic output resistance of the LNTA acts as resistor R for the N-path filter and as an $\mathrm{N}$-path filter is high-ohmic in-band, there will be significant voltage gain in-band $[55,56]$. The LNTA actually works as LNA with voltage gain in-band, but the voltage gain quickly drops out-of-band where the N-path filter is low-ohmic, and the desired LNTA behavior as linear V-I converter is realized (low output voltage swing). Hence improved linearity and compression are still possible at sufficient frequency offset from the N-path filter center frequency. On the other hand linearity is significantly worse than in passive-first receivers, although hybrid approaches are also possible, see e.g. [25, 58]. Overall, significant power consumption savings are certainly possible, as the N-path filter switches can be much smaller than in a 50ohm environment, see for instance the power comparison in [59].

An alternative approach is shown in Fig. 10b, known as "gainboosted N-path filter" [36] or as "Miller Bandpass Filter" [54]. Here an N-path notch-filter [17] is put in the feedback path of a voltage amplifying LNA, consisting of transconductance $\mathrm{G}_{\mathrm{m}}$ and load resistance $\mathrm{R}_{\mathrm{L}}$. The lack of feedback at the notchfrequency leads to a peak-gain of the total negative feedback circuit, i.e. an LNA with band-pass filtering. The input signal $\mathrm{V}_{\mathrm{i}}$ is filtered now by an N-path filter, but in a "gain-boosted" way or benefiting from the Miller effect, which has two key advantages:

1) Due to the Miller effect, the feedback capacitors see $\left(\mathrm{A}_{\mathrm{v}}+1\right)$ times more voltage than the input voltage and the $\mathrm{N}$ path filter impedance seen from the input is lowered by this factor. Hence $\left(\mathrm{A}_{\mathrm{v}}+1\right)$ times less capacitance is needed.

2) For the same N-path filter attenuation and noise, the on-resistance of the switches can be increased by a factor

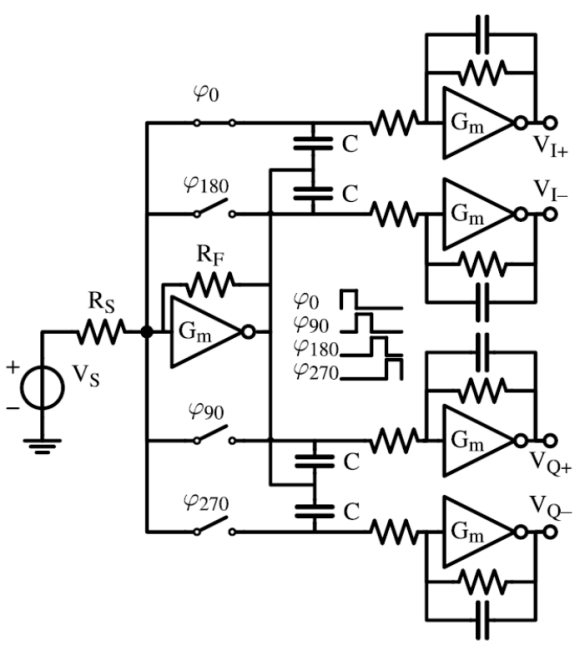

Fig. 12. Gain-Boosted 4-Path with including downconversion mixing [61].

$\left(\mathrm{A}_{\mathrm{v}}+1\right)$, reducing switch size and saving considerable dynamic power consumption in the drivers of the switch transistors.

This concept of a BPF-LNA, due to a notch filter in the feedback, can be enhanced further by using the capacitive $\pi$ network shown in Fig. 11 [60]. This introduces extra notches useful for TX-leakage suppression. Combining this technique with large overdrive voltage for the switches using thick-oxide transistors in $32 \mathrm{~nm}$ SOI CMOS technology, an out-of-band IIP3 of $+36 \mathrm{dBm}$ and $\mathrm{B} 1 \mathrm{~dB}$ of $+17 \mathrm{dBm}$ is achieved.

An interesting recent idea that targets low power combines the gain boosted N-path filtering in the LNA with frequency down-conversion as shown in Fig. 12. As the switched-C notch-filter capacitors contain baseband information, this can be sensed differentially and low-pass filtered without additional switches, leading to a compact low power receiver [61]. Other papers target ultra-low-power N-path filters by increasing impedance level and using only three clock phases [62] or even only two phases, if phase information and image rejection is irrelevant [63].

\section{DiscusSiON AND NEW APPLICATION DiRECTIONS}

As discussed above, N-path filtering can bring flexibility and high linearity. To benchmark the achieved results, Fig. 13 shows out-of-band IIP3 versus offset frequency normalized to $-3 \mathrm{~dB}$ bandwidth. Not surprisingly, the best linearity comes at a price in Noise Figure (see labels in Fig. 13). As might be expected, the compact power-efficient concepts Fig. 10 achieve modest linearity $(\sim 10 \mathrm{dBm})$ because the non-linear properties of transconductors degrade the linearity performance compared to what is possible with passive switchR-C circuits. As different applications require different performance, there seems place for multiple concepts.

Apart from software defined radio applications, other applications are also explored. Below we name a few. N-path filters can not only be used for frequency-domain filtering, but also for spatial domain filtering, i.e. beamforming, as demonstrated in [55, 59, 64-66]. Especially if implemented at the first node of the receiver, very high compression point and 


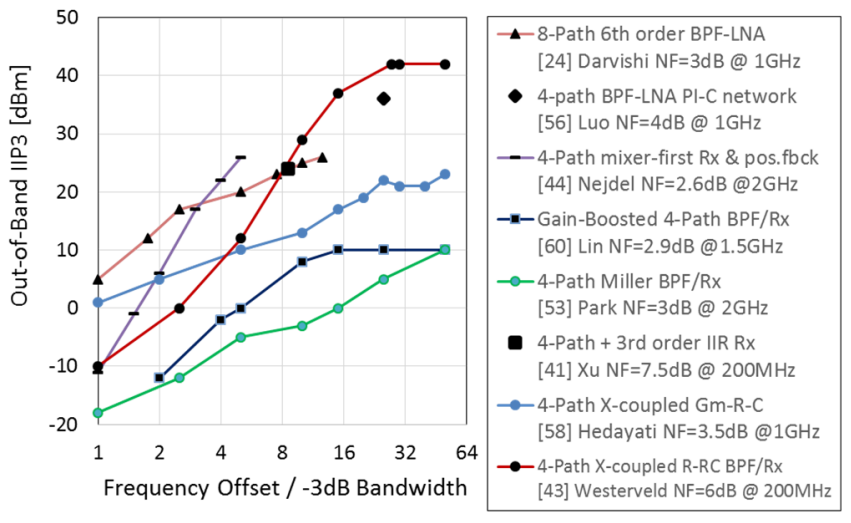

Fig. 13. Comparison of IIP3 versus offset frequency normalized to $-3 \mathrm{~dB}$ bandwidth for different publications. NF and test frequency are indicated.

IIP3 is possible [66]. Another interesting property of N-path filtering is the controllable gain and group delay [67], which was exploited to improve wideband self-interference cancellation for an in-channel full duplex transceiver [68]. Intriguingly, N-path filters can show non-reciprocal behavior allowing the realization of circulator-like behavior [69].

\section{CONCLUSIONS}

During the last decade N-path filtering and mixer-first receivers attracted a lot of research interest, as highly linear high-Q filtering is feasible around a frequency that is digitally accurately controllable. Very promising performance has been demonstrated in the low $\mathrm{GHz}$ range, e.g. blocker compression points $>+10 \mathrm{dBm}$, Out-of-band IIP3 $>40 \mathrm{dBm}$ and a $0 \mathrm{dBm}$ blocker noise figure $<5 \mathrm{~dB}$. Key challenges that remain are the realization of low phase noise multi-phase clocks at acceptable power consumption, and the implementation of sufficient prefiltering to limit folding adequately.

\section{REFERENCES}

[1] N. F. Barber, "Narrow Band-Pass Filter Using Modulation," Wireless Engineer, pp. 132-134, 1947.

[2] B. D. Smith, "Analysis of Commutated Networks," Aeronautical and Navigational Electronics, Transactions of the IRE Professional Group on IRE Trans., vol. PGAE-10, pp. 21-26, December 1953.

[3] W. R. LePage, C. R. Cahn, and J. S. Brown, "Analysis of a comb filter using synchronously commutated capacitors," American Institute of Electrical Engineers, Part I: Communication and Electronics, Transactions of the, vol. 72, pp. 63-68, March 1953.

[4] L. Franks and F. Witt, "Solid-state sampled-data bandpass filters," in SolidState Circuits Conference. Digest of Technical Papers. 1960 IEEE International, 1960 , pp. 70-71.

[5] L. E. Franks and I. W. Sandberg, "An Alternative Approach to the Realization of Network Transfer Functions: The N-Path Filters," Bell Sys. Tech. $J$., vol. 39, pp. 1321-1350, 1960.

[6] G. S. Moschytz, "Inductorless filters: a survey II. Linear active and digital filters," IEEE Spectrum, vol. 7, pp. 63-75, 1970.

[7] J. Zhu, H. Krishnaswamy, and P. R. Kinget, "Field-Programmable LNAs With Interferer-Reflecting Loop for Input Linearity Enhancement," IEEE Journal of Solid-State Circuits, vol. 50, pp. 556-572, 2015.

[8] D. Murphy, H. Darabi, A. Abidi, A. A. Hafez, A. Mirzaei, M. Mikhemar, et al., "A Blocker-Tolerant, Noise-Cancelling Receiver Suitable for Wideband Wireless Applications," Solid-State Circuits, IEEE Journal of, vol. 47, pp. 2943-2963, 2012.
[9] B. Razavi, "The role of translational circuits in RF receiver design," in Proceedings of the IEEE 2014 Custom Integrated Circuits Conference, 2014, pp. 1-8.

[10] B. Razavi, "Recent developments in RF receivers," in Proceedings of the IEEE 2014 Custom Integrated Circuits Conference, 2014, pp. 1-78.

[11]E. Klumperink and A. Molnar, "Interference Robust, Flexible Radio Receivers in CMOS," Radio Frequency Integrated Circuit Virtual Journal (IEEE), pp. available on-line: http://ieeexplore.ieee.org/xpls/virtualjournal/virtualJournalHome?pub=rfic, Jan 2016.

[12] A. Ghaffari, E. A. M. Klumperink, and B. Nauta, "A differential 4-path highly linear widely tunable on-chip band-pass filter," in Radio Frequency Integrated Circuits Symposium (RFIC), 2010 IEEE, 2010, pp. 299-302.

[13]E. Langer, "A new type of N-path N filters with two pairs of complex poles," in Solid-State Circuits Conference. Digest of Technical Papers. 1968 IEEE International, 1968, pp. 26-27.

[14]D. C. von Grunigen, R. P. Sigg, J. Schmid, G. S. Moschytz, and H. Melchior, "An integrated CMOS switched-capacitor bandpass filter based on N-path and frequency-sampling principles," Solid-State Circuits, IEEE Journal of, vol. 18, pp. 753-761, 1983.

[15] A. El Oualkadi, M. El Kaamouchi, J. M. Paillot, D. Vanhoenacker-Janvier, and D. Flandre, "Fully Integrated High-Q Switched Capacitor Bandpass Filter with Center Frequency and Bandwidth Tuning," in Radio Frequency Integrated Circuits (RFIC) Symposium, 2007, pp. 681-684.

[16] M. C. M. Soer, E. A. M. Klumperink, P. T. de Boer, F. E. van Vliet, and B. Nauta, "Unified Frequency-Domain Analysis of Switched-Series-RC Passive Mixers and Samplers," Circuits and Systems I: Regular Papers, IEEE Transactions on, vol. 57, pp. 2618-2631, 2010.

[17] A. Ghaffari, E. A. M. Klumperink, M. C. M. Soer, and B. Nauta, "Tunable High-Q N-Path Band-Pass Filters: Modeling and Verification," Solid-State Circuits, IEEE Journal of, vol. 46, pp. 998-1010, 2011.

[18] A. Mirzaei, H. Darabi, A. Yazdi, Z. Zhimin, E. Chang, and P. Suri, "A 65 nm CMOS Quad-Band SAW-Less Receiver SoC for GSM/GPRS/EDGE," Solid-State Circuits, IEEE Journal of, vol. 46, pp. 950-964, 2011.

[19] A. Mirzaei, H. Darabi, and D. Murphy, "A Low-Power Process-Scalable Super-Heterodyne Receiver With Integrated High-Q Filters," Solid-State Circuits, IEEE Journal of, vol. 46, pp. 2920-2932, 2011.

[20] A. Mirzaei and H. Darabi, "Analysis of Imperfections on Performance of 4-Phase Passive-Mixer-Based High-Q Bandpass Filters in SAW-Less Receivers," Circuits and Systems I: Regular Papers, IEEE Transactions on, vol. 58, pp. 879-892, 2011.

[21] A. Mirzaei, H. Darabi, and D. Murphy, "Architectural Evolution of Integrated M-Phase High-Q Bandpass Filters," Circuits and Systems I: Regular Papers, IEEE Transactions on, vol. 59, pp. 52-65, 2012.

[22] M. Darvishi, R. van der Zee, E. A. M. Klumperink, and B. Nauta, "Widely Tunable 4th Order Switched Gm-C Band-Pass Filter Based on N-Path Filters," Solid-State Circuits, IEEE Journal of, vol. 47, pp. 3105-3119, 2012.

[23] A. Ghaffari, E. A. M. Klumperink, and B. Nauta, "Tunable N-Path Notch Filters for Blocker Suppression: Modeling and Verification," Solid-State Circuits, IEEE Journal of, vol. 48, pp. 1370-1382, 2013.

[24]M. Darvishi, R. van der Zee, and B. Nauta, "Design of Active N-Path Filters," Solid-State Circuits, IEEE Journal of, vol. 48, pp. 2962-2976, 2013.

[25]D. Murphy, A. Mirzaei, H. Darabi, M. C. F. Chang, and A. Abidi, "An LTV Analysis of the Frequency Translational Noise-Cancelling Receiver," Circuits and Systems I: Regular Papers, IEEE Transactions on, vol. 61, pp. 266-279, 2014.

[26] B. W. Cook, A. Berny, A. Molnar, S. Lanzisera, and K. S. J. Pister, "LowPower 2.4-GHz Transceiver With Passive RX Front-End and 400-mV Supply," Solid-State Circuits, IEEE Journal of, vol. 41, pp. 2757-2766, 2006.

[27] M. Soer, E. A. M. Klumperink, Z. Ru, F. E. van Vliet, and B. Nauta, "A 0.2 -to-2.0 GHz $65 \mathrm{~nm}$ CMOS receiver without LNA achieving $>11 \mathrm{dBm}$ IIP3 and $<6.5 \mathrm{~dB}$ NF," in Solid-State Circuits Conference - Digest of Technical Papers, 2009. ISSCC 2009. IEEE International, 2009, pp. 222-223,223a.

[28]C. Andrews and A. C. Molnar, "Implications of Passive Mixer Transparency for Impedance Matching and Noise Figure in Passive Mixer-First Receivers," Circuits and Systems I: Regular Papers, IEEE Transactions on, vol. 57, pp. 3092-3103, 2010.

[29] C. Andrews and A. C. Molnar, "A Passive Mixer-First Receiver With Digitally Controlled and Widely Tunable RF Interface," Solid-State Circuits, IEEE Journal of, vol. 45, pp. 2696-2708, 2010.

[30] A. Molnar and C. Andrews, "Impedance, filtering and noise in n-phase passive CMOS mixers," in Custom Integrated Circuits Conference (CICC), 2012 IEEE, 2012, pp. 1-8. 
[31]C. Andrews, L. Changhyuk, and A. Molnar, "Effects of LO harmonics and overlap shunting on N-phase passive mixer based receivers," in ESSCIRC (ESSCIRC), 2012 Proceedings of the, 2012, pp. 117-120.

[32] C. Andrews, L. Diamente, D. Yang, B. Johnson, and A. Molnar, "A Wideband Receiver With Resonant Multi-Phase LO and Current Reuse Harmonic Rejection Baseband," Solid-State Circuits, IEEE Journal of, vol. 48, pp. 1188-1198, 2013.

[33] T. Iizuka and A. A. Abidi, "FET-R-C Circuits: A Unified Treatment - Part II: Extension to Multi-Paths, Noise Figure, and Driving-Point Impedance," IEEE Transactions on Circuits and Systems I: Regular Papers, vol. 63, pp. 1337-1348, 2016

[34] T. Iizuka and A. A. Abidi, "FET-R-C Circuits: A Unified Treatment - Part I: Signal Transfer Characteristics of a Single-Path," IEEE Transactions on Circuits and Systems I: Regular Papers, vol. 63, pp. 1325-1336, 2016.

[35] E. A. M. Klumperink, M. C. M. Soer, R. Struiksma, F. E. v. Vliet, and B. Nauta, Towards Low Power N-Path Filters for Flexible RF-Channel Selection. Switzerland: Springer International Publishing, 2015.

[36]Z. Lin, P. I. Mak, and R. P. Martins, "Analysis and Modeling of a GainBoosted N-Path Switched-Capacitor Bandpass Filter," Circuits and Systems I: Regular Papers, IEEE Transactions on, vol. PP, pp. 1-9, 2014.

[37] L. Duipmans, R. E. Struiksma, E. A. M. Klumperink, B. Nauta, and F. E. van Vliet, "Analysis of the Signal Transfer and Folding in N-Path Filters With a Series Inductance," Circuits and Systems I: Regular Papers, IEEE Transactions on, vol. 62, pp. 263-272, 2015.

[38] S. Hameed, M. Rachid, B. Daneshrad, and S. Pamarti, "Frequency-Domain Analysis of N-Path Filters Using Conversion Matrices," IEEE Transactions on Circuits and Systems II: Express Briefs, vol. 63, pp. 74-78, 2016.

[39] D. Yang, C. Andrews, and A. Molnar, "Optimized Design of N-Phase Passive Mixer-First Receivers in Wideband Operation," IEEE Transactions on Circuits and Systems I: Regular Papers, vol. 62, pp. 2759-2770, 2015.

[40]A. Mirzaei, H. Darabi, J. C. Leete, and C. Yuyu, "Analysis and Optimization of Direct-Conversion Receivers With 25\% Duty-Cycle CurrentDriven Passive Mixers," Circuits and Systems I: Regular Papers, IEEE Transactions on, vol. 57, pp. 2353-2366, 2010

[41] Y. Xu and P. R. Kinget, "A Switched-Capacitor RF Front End With Embedded Programmable High-Order Filtering," IEEE Journal of Solid-State Circuits, vol. 51, pp. 1154-1167, 2016.

[42] S. Jayasuriya, Y. Dong, and A. Molnar, "A baseband technique for automated LO leakage suppression achieving $<-80 \mathrm{dBm}$ in wideband passive mixer-first receivers," in Custom Integrated Circuits Conference (CICC), 2014 IEEE Proceedings of the, 2014, pp. 1-4.

[43] C. Wu, Y. Wang, B. Nikolić, and C. Hull, "An Interference-Resilient Wideband Mixer-First Receiver With LO Leakage Suppression and I/Q Correlated Orthogonal Calibration," IEEE Transactions on Microwave Theory and Techniques, vol. 64, pp. 1088-1101, 2016.

[44] H. Westerveld, E. Klumperink, and B. Nauta, "A cross-coupled switch-RC mixer-first technique achieving $+41 \mathrm{dBm}$ out-of-band IIP3," in 2016 IEEE Radio Frequency Integrated Circuits Symposium (RFIC), 2016, pp. 246-249.

[45]A. Nejdel, M. Abdulaziz, T. M, and H. Sjöland, "A positive feedback passive mixer-first receiver front-end," in Radio Frequency Integrated Circuits Symposium (RFIC), 2015 IEEE, 2015, pp. 79-82.

[46] A. Mirzaei, H. Darabi, J. C. Leete, C. Xinyu, K. Juan, and A. Yazdi, "Analysis and Optimization of Current-Driven Passive Mixers in Narrowband Direct-Conversion Receivers," Solid-State Circuits, IEEE Journal of, vol. 44, pp. 2678-2688, 2009.

[47] R. E. Struiksma, E. A. M. Klumperink, B. Nauta, and F. E. van Vliet, "A $500 \mathrm{MHz}-2.7 \mathrm{GHz} 8$-path weaver downconverter with harmonic rejection and embedded filtering," in European Solid State Circuits Conference (ESSCIRC), ESSCIRC 2014 - 40th, 2014, pp. 223-226.

[48] W. Heinlein, K. Moehrmann, and W. Holmes, "Double-tuned N-path bandpass filters using a single gyrator," Circuit Theory, IEEE Transactions on, vol. 18, pp. 728-729, 1971.

[49]R. Chen and H. Hashemi, "Reconfigurable Receiver With RadioFrequency Current-Mode Complex Signal Processing Supporting Carrier Aggregation," Solid-State Circuits, IEEE Journal of, vol. 50, pp. 3032-3046, 2015 .

[50] C. M. Thomas and L. E. Larson, "Broadband Synthetic Transmission-Line N-Path Filter Design," IEEE Transactions on Microwave Theory and Techniques, vol. 63, pp. 3525-3536, 2015.

[51]C. k. Luo, P. S. Gudem, and J. F. Buckwalter, "A 0.2 -3.6-GHz 10-dBm B1dB 29-dBm IIP3 Tunable Filter for Transmit Leakage Suppression in SAW-
Less 3G/4G FDD Receivers," IEEE Transactions on Microwave Theory and Techniques, vol. 63, pp. 3514-3524, 2015.

[52] C. k. Luo and J. F. Buckwalter, "A 0.25-to-2.25 GHz, 27 dBm IIP3, 16Path Tunable Bandpass Filter," IEEE Microwave and Wireless Components Letters, vol. 24, pp. 866-868, 2014

[53] J. Borremans, G. Mandal, V. Giannini, B. Debaillie, M. Ingels, T. Sano, et al., "A 40 nm CMOS 0.4-6 GHz Receiver Resilient to Out-of-Band Blockers," Solid-State Circuits, IEEE Journal of, vol. 46, pp. 1659-1671, 2011.

[54] J. W. Park and B. Razavi, "Channel Selection at RF Using Miller Bandpass Filters," Solid-State Circuits, IEEE Journal of, vol. 49, pp. 3063-3078, 2014.

[55] M. C. M. Soer, E. A. M. Klumperink, B. Nauta, and F. E. van Vliet, "Spatial Interferer Rejection in a Four-Element Beamforming Receiver FrontEnd With a Switched-Capacitor Vector Modulator," Solid-State Circuits, IEEE Journal of, vol. 46, pp. 2933-2942, 2011.

[56]F. Qazi, Q. T. Duong, and J. Dabrowski, "Two-Stage Highly Selective Receiver Front-End Based on Impedance Transformation Filtering," Circuits and Systems II: Express Briefs, IEEE Transactions on, vol. PP, pp. 1-1, 2014.

[57] H. Hedayati, W. F. A. Lau, N. Kim, V. Aparin, and K. Entesari, "A 1.8 dB NF Blocker-Filtering Noise-Canceling Wideband Receiver With Shared TIA in $40 \mathrm{~nm}$ CMOS," IEEE Journal of Solid-State Circuits, vol. 50, pp. 1148-1164, 2015 .

[58] H. Hedayati, V. Aparin, and K. Entesari, "A +22dBm IIP3 and 3.5dB NF wideband receiver with RF and baseband blocker filtering techniques," in 2014 Symposium on VLSI Circuits Digest of Technical Papers, 2014, pp. 1-2.

[59] M. C. M. Soer, E. A. M. Klumperink, B. Nauta, and F. E. van Vliet, "A 1.0 -to- $2.5 \mathrm{GHz}$ beamforming receiver with constant-Gm vector modulator consuming $<9 \mathrm{~mW}$ per antenna element in 65nm CMOS," in Solid-State Circuits Conference Digest of Technical Papers (ISSCC), 2014 IEEE International, 2014, pp. 66-67.

[60] C. k. Luo, P. S. Gudem, and J. F. Buckwalter, "0.4 - 6 GHz,17-dBm B1dB, 36-dBm IIP3 channel-selecting, low-noise amplifier for SAW-less 3G/4G FDD receivers," in Radio Frequency Integrated Circuits Symposium (RFIC), 2015 IEEE, 2015, pp. 299-302.

[61] L. Zhicheng, M. Pui-In, and R. P. Martins, "2.4 A $0.028 \mathrm{~mm}^{2} 11 \mathrm{~mW}$ singlemixing blocker-tolerant receiver with double-RF N-path filtering, $\mathrm{S}_{11}$ centering, $+13 \mathrm{dBm}$ OB-IIP3 and 1.5-to-2.9dB NF," in Solid-State Circuits Conference(ISSCC), 2015 IEEE International, 2015, pp. 1-3.

[62]R. Dutta, R. v. d. Zee, A. B. J. Kokkeler, M. J. Bentum, E. A. M Klumperink, and B. Nauta, "An Ultra Low Energy FSK Receiver With In-Band Interference Robustness Exploiting a Three-Phase Chirped LO," IEEE Journal on Emerging and Selected Topics in Circuits and Systems, vol. 4, pp. 248-261, 2014.

[63] C. Salazar, A. Cathelin, A. Kaiser, and J. Rabaey, "A 2.4 GHz InterfererResilient Wake-Up Receiver Using A Dual-IF Multi-Stage N-Path Architecture," IEEE Journal of Solid-State Circuits, vol. 51, pp. 2091-2105, 2016.

[64] M. C. M. Soer, E. A. M. Klumperink, B. Nauta, and F. E. van Vliet, "A 1.0-to-4.0GHz $65 \mathrm{~nm}$ CMOS four-element beamforming receiver using a switched-capacitor vector modulator with approximate sine weighting via charge redistribution," in Solid-State Circuits Conference Digest of Technical Papers (ISSCC), 2011 IEEE International, 2011, pp. 64-66.

[65] M. Soer, E. Klumperink, B. Nauta, and F. van Vliet, "A 1.5-to-5.0GHz input-matched $+2 \mathrm{dBm} \mathrm{P} 1 \mathrm{~dB}$ all-passive switched-capacitor beamforming receiver front-end in 65nm CMOS," in Solid-State Circuits Conference Digest of Technical Papers (ISSCC), 2012 IEEE International, 2012, pp. 174-176.

[66] A. Ghaffari, E. A. M. Klumperink, F. van Vliet, and B. Nauta, "A 4Element Phased-Array System With Simultaneous Spatial- and FrequencyDomain Filtering at the Antenna Inputs," Solid-State Circuits, IEEE Journal of, vol. 49, pp. 1303-1316, 2014.

[67] N. Reiskarimian, J. Zhou, T. H. Chuang, and H. Krishnaswamy, "Analysis and Design of Two-Port N-Path Bandpass Filters With Embedded Phase Shifting," IEEE Transactions on Circuits and Systems II: Express Briefs, vol. 63, pp. 728-732, 2016

[68] J. Zhou, N. Reiskarimian, and H. Krishnaswamy, "9.8 Receiver with integrated magnetic-free N-path-filter-based non-reciprocal circulator and baseband self-interference cancellation for full-duplex wireless," in 2016 IEEE International Solid-State Circuits Conference (ISSCC), 2016, pp. 178-180.

[69] N. Reiskarimian and H. Krishnaswamy, "Magnetic-free non-reciprocity based on staggered commutation," Nature Communications, vol. 7, p. 11217 , 04/15/online 2016. 